\title{
Serum neopterin concentrations and tryptophan degradation pattern in patients with late stage larynx carcinoma
}

DOI 10.1515/pterid-2017-0004

Received March 10, 2017; accepted May 16, 2017; previously published online June 30, 2017

\begin{abstract}
As the disease-free 5-year-survival of late stage laryngeal carcinoma patients is extremely low, indoleamine-2,3-dioxygenase-1 (IDO)-induced tryptophan degradation may represent an immune escape mechanism which plays an important role in cancer spreading in advanced stage laryngeal cancers. We examined whether the late stage laryngeal cancer enhances tumor immune evasion by the expression of systemic IDO activities and chronic cellular immune activation. Twenty-two of 42 male laryngeal cancer patients were classified as late stage cancer according to American Joint Committee on Cancer (AJCC) criteria. Their serum neopterin, tryptophan and kynurenine concentrations were compared with 30 cancer-free individuals. IDO activity was approved by correlation between serum neopterin and kynurenine/tryptophan. Late stage cancer patients preoperatively showed a significantly higher IDO activity compared to controls and early stage cancer cases. Six months after tumor removal, late stage cancer patients although having higher serum neopterin concentration compared to early stage patients or controls, they showed a significant decrease in IDO activity and tryptophan consumption. Increased systemic IDO activity may provoke the escape of tumor cells from the immune surveillance of the host. High IDO activity is due to the presence of tumor mass. Persistence of high serum neopterin levels despite tumor removal may indicate poor prognosis.
\end{abstract}

\footnotetext{
*Corresponding author: Ayse Basak Engin, Department of Toxicology, Faculty of Pharmacy, Gazi University, 06330 Hipodrom, Ankara, Turkey, E-mail: abengin@gmail.com

Riza Onder Gunaydin and Ali Sefik Hosal: Department of Otorhinolaryngoloy Head and Neck Surgery, Faculty of Medicine, Hacettepe University, Ankara, Turkey

Sacit Altug Kesikli: Department of Basic Immunology, Cancer Institute, Hacettepe University, Altindag, Ankara, Turkey Dietmar Fuchs: Division of Biological Chemistry, Biocenter, Innsbruck Medical University, Innsbruck, Austria
}

Keywords: indoleamine 2,3-dioxygenase; larynx cancer; neopterin; tryptophan.

\section{Introduction}

Head and neck squamous cell carcinoma is the sixth most common neoplasm in the world. Despite advances in treatments involving surgery, radiation, and chemotherapy, the 5 -year survival is $<50 \%$, because of local recurrence of this aggressive malignancy [1]. Although surgery is a favorable prognostic factor for late stage laryngeal cancer, it does not impact on overall survival [2]. In this respect, head and neck squamous cell carcinomas are highly immunogenic tumors in which tumor-infiltrating cells consist largely of CD4+ lymphocytes [3]. Using an antibody against CD4 is a potent strategy for eliminating immunosuppressive natural CD4+ Treg cells and inducing lasting immunity to cancer [4]. Head and neck cancers often induce profound immunosuppression which contributes to disease progression and interferes with immune-based therapies. Body fluids of head and neck cancer patients are enriched in exosomes potentially engaged in negative regulation of anti-tumor immune responses [5]. In this context, by considering the data obtained from clinical trials, immunological escape mechanisms and key immunotherapies are discussed in head and neck squamous cell carcinomas [6].

Tryptophan degradation rate by tumor cells and high serum IDO activity may be critical for the balance between host immune response and the capacity of the tumor to evade normal host immune defense [7]. In this respect, the proinflammatory cytokine, interferon-gamma (IFN- $\gamma$ ) is thought to be associated with anti-tumoral cellular immunity [8]. In fact, indoleamine 2,3-dioxygenase (IDO) is overexpressed in response to IFN- $\gamma$ in a variety of malignancies. This enzyme causes immunosuppression through breakdown of tryptophan (Trp) in the tumor and tumor-draining lymph nodes [9]. Additionally, another substance synthesized by activated monocytes/ macrophages in response to IFN- $\gamma$ is neopterin. Measurement of neopterin concentrations in body fluids provides 
information about $\mathrm{T}$ helper cell 1 (Th1)-derived cellular immune activation [10, 11]. Recently, although a number of clinical studies examined the role of IDO in the patients with cancer, we could not find any data considering larynx cancers [7, 12]. The disease-free 5-year-survival rate for patients suffering from late stage laryngeal carcinoma is between $32.4 \%$ and $6.7 \%$. The tumor size and free margins proved to be absolute significant prognostic factors [13]. Therefore, the immunosuppressive environment is of great importance for advanced stage laryngeal cancers. In this study, it was examined whether the late stage of laryngeal cancer enhances IDO activity and in accordance with this, alters the increased frequency of serum neopterin concentration.

\section{Materials and methods}

\section{Patients and methods}

In total, 42 male laryngeal squamous cell carcinoma patients with a median age of 58.5 years (range: 41-73 years) were included in this study. Thirty male cancer-free voluntary individuals with similar characteristics served as control. All participants' rights were protected and informed consent was obtained according to the Helsinki Declaration. Ethical approval was obtained from Hacettepe University, Medical Faculty Local Research Ethics Committee. Peripheral venous blood samples were collected from voluntary individuals and patients with histologically confirmed to have primary laryngeal squamous cell carcinoma, into anticoagulant free blood collection tube and used for the serum separation. All samples were obtained in the early morning, protected from direct light and kept in $-20^{\circ} \mathrm{C}$ until assayed. Patients had no other known malignancies, infections, or chronic diseases and had not received preoperative chemotherapy and/or radiotherapy. Age, tumor site, tumor stage, histological grade, total radiation dose and surgery margins of the patients were recorded. The patients were classified according to American Joint Committee on Cancer (AJCC), tumor-node-metastasis (TNM) classification; seven patients were stage 1,13 were stage 2, 10 were stage 3 and 12 were stage 4 . Afterwards 42 patients were allocated into two groups; early stage (stage 1 and 2, $\mathrm{n}=20$ ) and late stage (stage 3 and $4, n=22$ ).

Serum neopterin concentrations were determined by a commercially available enzyme immunoassay kit (ELISA, Demeditec Diagnostics, Kiel, Germany), according to the manufacturer's instructions. The optical density was measured at $450 \mathrm{~nm}$. Trp and kynurenine (Kyn) concentrations in serum were determined by reversed-phase high-pressure liquid chromatography following precipitation of protein with trichloroacetic acid. Trp was measured via fluorescence detection at $285 \mathrm{~nm}$ excitation wavelengths and $365 \mathrm{~nm}$ emission wavelengths, while Kyn was detected by UV absorption at the $360 \mathrm{~nm}$ wavelengths, simultaneously [14]. In order to determine the IDO activity, Kyn to Trp ratio (Kyn/Trp) was calculated by dividing Kyn concentrations $(\mathrm{nmol} / \mathrm{L})$ by $\operatorname{Trp}(\mu \mathrm{mol} / \mathrm{L})$, subsequently the correlation between the serum neopterin and Kyn/Trp ratio was examined [15]. The presence of IDO activity was approved in the case of significantly positive correlation between the serum neopterin and Kyn/Trp ratio.

\section{Statistical analysis}

Data were analyzed by using the statistical package SPSS, version 13.0 (SPSS Inc., Chicago, IL, USA). All results were expressed as the mean \pm standard error of mean (SEM). After checking the data by Kolmogorov-Smirnov test, non-parametric data of the two independent groups were compared with Mann-Whitney U-test and $\mathrm{p}<0.05$ was considered statistically significant. Correlations were assessed using Spearman's rank test.

\section{Results}

Preoperative neopterin concentrations in total cancer patients were significantly higher than that of cancerfree controls $(p=0.0001)$. Although the neopterin levels of early stage cancer patients and healthy controls were similar, late stage group had significantly higher neopterin compare to both control and early stage cancer individuals (Figure 1). While the increased frequency of neopterin concentrations was $15 \%$ in early cancer cases, at the late stage it was 59\%. Preoperative Trp degradation rate was significantly higher in late stage cancer patients. However, first detectable degradation product of Trp, Kyn was only slightly higher but not significantly different in late stage laryngeal cancer $(p=0.01$, late stage vs. control). The increases of neopterin and Kyn/Trp concentrations in the various subgroups of patients seemed to

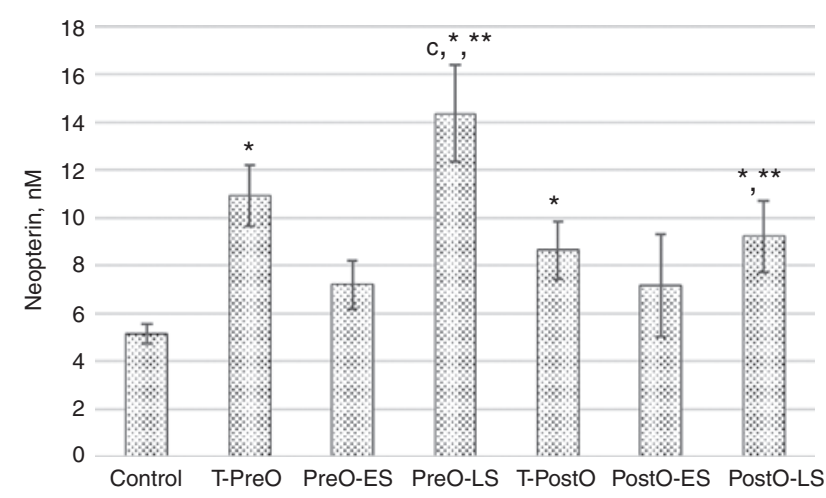

Figure 1: Alterations in the neopterin levels of the control and larynx cancer patient groups.

T, Total larynx cancer patients group; Pre0, pre-operative; PostO, post-operative; ES, early stage larynx cancer; LS, late stage larynx cancer; ${ }^{c} p<0.01$, Spearman correlation, PreO-LS neopterin PreO-LS Kyn/Trp; ${ }^{\star} p<0.01$, control vs. patient group; ${ }^{\star *} p<0.01$, ES vs. LS patient groups. 
develop in a very parallel manner. On the other hand, late stage cancer patients showed significantly higher Kyn/Trp ratio versus both control and early stage cancer individuals ( $p=0.0004$ and $p=0.0146$, respectively) (Figure 2). A highly significant correlation was observed between the preoperative values of neopterin and Kyn/Trp ratio in the late stage cancer group $(\mathrm{p}=0.0073, \mathrm{r}=+0.555)$ and it was recognized as enhanced IDO activity.

However, postoperative evaluation of laryngeal cancer patients revealed that the late stage group had still higher serum neopterin concentration when compared to early stage patients or controls. After the operation, despite the overall increased mean values of the groups, the frequencies of increased neopterin concentrations decreased to $5 \%$ and $14 \%$, respectively, for the early stage and late stage cancer patients. On the other hand, neither Trp nor Kyn levels showed a significant rise. Although the postoperative $\mathrm{Kyn} / \mathrm{Trp}$ ratio of the late stage carcinoma group showed a significant increase in comparison to controls, we did not observe a significant correlation with the neopterin levels. Surprisingly the lack of increase in postoperative IDO activity was attributed to the reduction of tumor tissue, even if not completely removed.

\section{Discussion}

Altered immune, inflammatory, and angiogenic responses have been noticed in head and neck cancer, and many of these responses have been associated with a poor clinical outcome [16]. Unfortunately, patients with head and

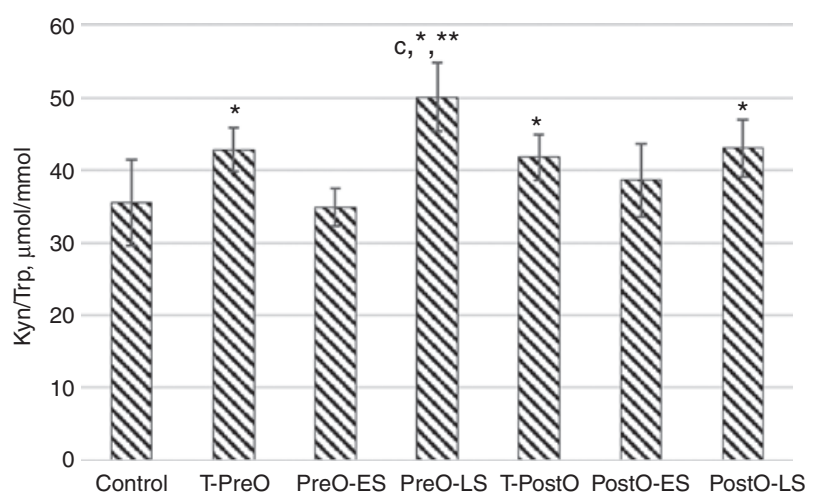

Figure 2: Alterations in the kynurenine/tryptophan levels of the control and larynx cancer patient groups.

T, Total larynx cancer patients group; Pre0, pre-operative; Post0, post-operative; ES, early stage larynx cancer; LS, late stage larynx cancer; ${ }^{c} p<0.01$, Spearman correlation, PreO-LS neopterin PreO-LS Kyn/Trp; ${ }^{\star} p<0.01$, control vs. patient group; ${ }^{\star \star} p<0.01$, ES vs. LS patient groups. neck squamous cell carcinoma have profound immune defects that are associated with increased recurrence [1]. Upon induction by Th1-type cytokine, IFN- $\gamma[17,18]$, tumors expressing IDO at a high level may effectively escape from the immune surveillance of the host by degrading local Trp [19]. Indeed, in cancer patients, degradation of Trp is significantly accelerated. In this case, serum Trp concentrations decrease; Kyn and Kyn to Trp ratio increases [20]. Increase in IDO activity and the accumulation of Trp metabolites results in a strong inhibitory effect on the development of immune responses by blocking $\mathrm{T}$ cell activation, inducing $\mathrm{T}$ cell apoptosis or promoting the regulatory $\mathrm{T}$ cell (Tregs) differentiation [21]. Kyn binding to human aryl hydrocarbon receptor (AHR) is essential to generate Tregs that suppress adaptive immunity [22]. Thereby, IDO-expressing cells have been considered to create a state of immunological unresponsiveness towards tumor-derived antigens [23]. In particular, the enzyme IDO has recently attracted special attention [24] and may be expressed constitutively by tumor cells as part of the genetic changes involved in malignant transformation $[19,25]$. Although IDO expression by tumor cells has been shown to be correlated with a poor clinical prognosis [15], there is no convincing data considering the degradation of Trp by IDO in laryngeal carcinoma cases. In our study, patients with late stage laryngeal cancer preoperatively showed significantly higher IDO activity $(p=0.0146)$ and $\operatorname{Trp}$ degradation pattern $(\mathrm{p}=0.0015)$ in contrast to the early stage patients. The presence of IDO activity was approved in the case of positively significant correlation between the serum neopterin and Kyn/Trp ratio. Thus, the highly significant correlation between neopterin concentrations with increased Kyn/Trp clearly indicated that the formation of Kyn is related to IDO activity by IFN- $\gamma$ stimulation in late stage laryngeal cancer cases. Surprisingly, late stage laryngeal carcinoma cases could not demonstrate higher IDO activity and excess Trp consumption following removal of tumor mass within 6-months period. Eventually, the correlation between neopterin and Kyn/ Trp ratio was disrupted. Thereby, it is claimed that tumororiginated IDO limits immune cell proliferation by depleting locally available Trp and/or producing its cytotoxic metabolites [26]. Fallarino et al. showed that IDO has been implicated in immune-tolerance because it can inhibit the immune response, either by exhausting L-Trp in the medium or producing toxic metabolites that can cause apoptosis in T cells [27].

On the other hand, an increase in neopterin concentrations during cancer growth indicates a chronic cellular immune response, but it is not specific for malignant cell proliferation [28]. If the presence of the tumor 
is previously confirmed by the tissue diagnosis, higher neopterin levels may be considered as cancer-related chronic immune activation. Nevertheless, increased frequency of neopterin levels among the advanced cancer cases was an evidence of enhanced macrophage response to tumor antigen. However, the neopterin levels of the patients with late stage laryngeal cancer were still higher postoperatively than the other groups, but it was found to be below the cut-off value. These results suggested that without considering the tumor margin, surgical removal of tumor mass reduces the Trp degradation and IDO activity but cannot further affect the cell-mediated immunity. Postoperative evaluation of serum neopterin levels in laryngeal cancer patients revealed that late stage group had still higher serum neopterin concentration when compared to early stage patients. High IDO activity results in the progression of tumor mass, persistence of high serum neopterin levels despite the tumor removal may suggest poor prognosis. Actually, the mean serum neopterin level in the non-tumor group is also below the standard cut-off value, $10 \mathrm{nmol} / \mathrm{L}$ [29]. Higher neopterin measurements not only provide an insight into the present state of cellmediated immune response but also allow monitoring and prognosis of disease progression [30]. Our results indicated that, patients with late stage cancer were still immunosuppressed, when compared to patients with non-metastatic disease, despite surgical removal of the tumor [31]. Furthermore, IDO inhibition may significantly enhance the antitumor activity of various chemotherapeutic and immunotherapeutic agents. These data are consistent with the results and showed that increased IDO expression is an independent prognostic variable for reduced overall survival in cancer patients [21]. Although the overall increase in postoperative neopterin levels of cancer cases is marked, in advanced cancer group increase in neopterin concentration is highly significant [32]. Increased neopterin concentrations associate with the endogenously formed IFN- $\gamma$, which is released as a result of the host-tumor interaction [33, 34]. While the increase of neopterin reflects the activation of monocytederived cells like macrophages or dendritic cells [35], the changes of IDO activity may indicate the possible contribution of tumor cells to Trp metabolism.

\section{Conclusion}

Increase in serum IDO activities of late stage laryngeal squamous cell carcinoma patients causes serious metabolic alterations which may facilitate the tumor progression irrespective of histopathological parameters. This study showed that besides the clinical manifestations, identification of systemic IDO activity may provide a preliminary information at diagnosis, regarding tumor stage in patients with laryngeal cancer. While, tumor removal in advanced cases results in diminished IDO activity, persistent serum neopterin concentration indicates poor prognosis.

Acknowledgements: This study has been orally presented at 32nd Winter-Workshop on Clinical, Chemical and Biochemical Aspects of Pteridines, February 23-March 03, 2013, St Christoph/Arlberg, Austria.

Conflict of interest statement: All authors have declared no conflicts of interest. All authors contributed to the manuscript and approved its final version.

\section{References}

1. Young MRI. Protective mechanisms of head and neck squamous cell carcinomas from immune assault. Head Neck 2006;28: 462-70.

2. Nguyen-Tan PF, Le QT, Quivey JM, Singer M, Terris DJ, Goffinet DR, et al. Treatment results and prognostic factors of advanced T3-4 laryngeal carcinoma: the University of California, San Francisco (UCSF) and Stanford University Hospital (SUH) experience. Int J Radiat Oncol Biol Phys 2001;50:1172-80.

3. Vitolo D, Gallo A, Ciocci L, Cicerone E, Baroni CD. Interleukin-12 related cytokine gene expression at a tissue level in carcinomas of the larynx. Eur Arch Otorhinolaryngol 2000;257:290-4.

4. Côté AL, Byrne KT, Steinberg SM, Zhang P, Turk MJ. Protective CD8 memory $T$ cell responses to mouse melanoma are generated in the absence of CD4 T cell help. PLoS One 2011;6:e26491.

5. Ludwig S, Floros T, Theodoraki MN, Hong CS, Jackson EK, Lang S, et al. Suppression of lymphocyte functions by plasma exosomes correlates with disease activity in patients with head and neck cancer. Clin Cancer Res 2017 Apr 11. pii: clincanres.2819.2016.

6. Xie X, O’Neill W, Pan Q. Immunotherapy for head and neck cancer: the future of treatment? Expert Opin Biol Ther 2017;17:1-8.

7. Engin AB, Ozkan Y, Fuchs D, Yardim-Akaydin S. Increased tryptophan degradation in patients with bronchus carcinoma. Eur J Cancer Care (Engl) 2010;19:803-8.

8. Conti-Freitas LC, Foss-Freitas MC, Mamede RC, Foss NT. Interferon-gamma and interleukin-10 production by mononuclear cells from patients with advanced head and neck cancer. Clin Sao Paulo Braz 2012;67:587-90.

9. Soliman H, Mediavilla-Varela M, Antonia S. Indoleamine 2,3-dioxygenase: is it an immune suppressor? Cancer J Sudbury Mass 2010;16:354-9.

10. Murr C, Bergant A, Widschwendter M, Heim K, Schröcksnadel $\mathrm{H}$, Fuchs D. Neopterin is an independent prognostic variable in females with breast cancer. Clin Chem 1999;45:1998-2004.

11. Murr C, Berchtold J, Norer B, Waldhart E, Wachter H, Fuchs D. Neopterin as a prognostic parameter in patients with squamouscell carcinomas of the oral cavity. Int J Cancer 1998;79:476-80. 
12. Engin A, Gonul II, Engin AB, Karamercan A, Sepici Dincel A, Dursun $A$. Relationship between indoleamine 2,3-dioxygenase activity and lymphatic invasion propensity of colorectal carcinoma. World J Gastroenterol 2016;22:3592-601.

13. Psychogios G, Waldfahrer F, Bozzato A, Iro H. Evaluation of the revised TNM classification in advanced laryngeal cancer. Eur Arch Otorhinolaryngol 2010;267:117-21.

14. Widner B, Werner ER, Schennach H, Wachter H, Fuchs D. Simultaneous measurement of serum tryptophan and kynurenine by HPLC. Clin Chem 1997;43:2424-6.

15. Brandacher G, Perathoner A, Ladurner R, Schneeberger S, Obrist $\mathrm{P}$, Winkler $\mathrm{C}$, et al. Prognostic value of indoleamine 2,3-dioxygenase expression in colorectal cancer: effect on tumor-infiltrating T cells. Clin Cancer Res 2006;12:1144-51.

16. Melinceanu L, Lerescu L, Tucureanu C, Caras I, Pitica R, Sarafoleanu C, et al. Serum perioperative profile of cytokines in patients with squamous cell carcinoma of the larynx. J Otolaryngol Head Neck Surg 2011;40:143-50.

17. Werner-Felmayer G, Werner ER, Fuchs D, Hausen A, Reibnegger $\mathrm{G}$, Wachter H. Characteristics of interferon induced tryptophan metabolism in human cells in vitro. Biochim Biophys Acta 1989;1012:140-7.

18. Yasui H, Takai K, Yoshida R, Hayaishi O. Interferon enhances tryptophan metabolism by inducing pulmonary indoleamine 2,3-dioxygenase: its possible occurrence in cancer patients. Proc Natl Acad Sci USA 1986;83:6622-6.

19. Uyttenhove C, Pilotte L, Théate I, Stroobant V, Colau D, Parmentier N, et al. Evidence for a tumoral immune resistance mechanism based on tryptophan degradation by indoleamine 2,3-dioxygenase. Nat Med 2003;9:1269-74.

20. Huang A, Fuchs D, Widner B, Glover C, Henderson DC, Allen-Mersh TG. Serum tryptophan decrease correlates with immune activation and impaired quality of life in colorectal cancer. Br J Cancer 2002;86:1691-6.

21. Liu X, Newton RC, Friedman SM, Scherle PA. Indoleamine 2,3-dioxygenase, an emerging target for anti-cancer therapy. Curr Cancer Drug Targets 2009;9:938-52.

22. Mezrich JD, Fechner JH, Zhang X, Johnson BP, Burlingham WJ, Bradfield CA. An interaction between kynurenine and the aryl hydrocarbon receptor can generate regulatory T cells. I Immunol 2010;185:3190-8.
23. Mellor AL, Munn DH. Tryptophan catabolism and T-cell tolerance: immunosuppression by starvation? Immunol Today 1999;20:469-73.

24. Zamanakou M, Germenis AE, Karanikas V. Tumor immune escape mediated by indoleamine 2,3-dioxygenase. Immunol Lett 2007;111:69-75.

25. Muller AJ, DuHadaway JB, Donover PS, Sutanto-Ward E, Prendergast GC. Inhibition of indoleamine 2,3-dioxygenase, an immunoregulatory target of the cancer suppression gene Bin1, potentiates cancer chemotherapy. Nat Med 2005;11:312-9.

26. Moffett JR, Namboodiri MA. Tryptophan and the immune response. Immunol Cell Biol 2003;81:247-65.

27. Fallarino F, Grohmann U, Vacca C, Bianchi R, Orabona C, Spreca A, et al. T cell apoptosis by tryptophan catabolism. Cell Death Differ 2002;9:1069-77.

28. Fuchs D, Hausen A, Reibnegger G, Werner ER, Dierich MP, Wachter H. Neopterin as a marker for activated cell-mediated immunity: application in HIV infection. Immunol Today 1988;9:150-5.

29. Wachter H, Fuchs D, Hausen A, Reibnegger G, Weiss G, Werner E, et al. Neopterin, biochemistry, methods, clinical application. Berlin: Walter de Gruyter, 1992.

30. Berdowska A, Zwirska-Korczala K. Neopterin measurement in clinical diagnosis. J Clin Pharm Ther 2001;26:319-29.

31. Kleinknecht S, Bichler KH, Strohmaier WL. Postoperative longterm course of peripheral blood immune parameters and immunomodulating effects of keyhole limpet hemocyanin in patients with nonmetastatic renal cell carcinoma. Eur Urol 1992;21:315-22.

32. Engin AB, Engin A, Sepici-Dincel A. Influence of surgical trauma on neopterin concentrations in gastric carcinoma patients. Tumori 2010;96:589-93.

33. Brandacher G, Winkler C, Schroecksnadel K, Margreiter R, Fuchs D. Antitumoral activity of interferon-gamma involved in impaired immune function in cancer patients. Curr Drug Metab 2006;7:599-612.

34. Yoshida R, Park SW, Yasui H, Takikawa O. Tryptophan degradation in transplanted tumor cells undergoing rejection. J Immunol 1988;141:2819-23.

35. Wirleitner B, Reider D, Ebner S, Böck G, Widner B, Jaeger M, et al. Monocyte-derived dendritic cells release neopterin. J Leukoc Biol 2002;72:1148-53. 